\section{Tribune libre Freies Forum}

\section{4e Symposium de printemps de I"EADV}

Saariselkä, du 9 au 12 février 2006

La Laponie en hiver? Quelle idée... Le Père Noël vient à nous en décembre (pour le Tarmed cela n'a pas été évident...), à quoi bon aller lui rendre visite dans la nuit et le froid polaires? La semaine précédant le congrès de l'EADV, le thermomètre ne s'était-il pas figé à $-28^{\circ} \mathrm{C}$ ?

Et pourtant...

Après un voyage plein de heurts et d'imprévus, nous voici sans bagages à Ivalo. Il ne fait que $-10^{\circ} \mathrm{C}$, tout va bien. Il ne fait pas nuit, mais une clarté laiteuse, magnifiée par la pleine lune.

Pas de bagages? Qu'importe! Les organisateurs ont tout prévu. Equipement arctique complet, qui nous permet de partir en motoneige à la pêche sur un lac glacé, une belle bredouille que nous font oublier ballades en raquettes et courses de traîneaux avec des huskies déchaînés. Le tout sous une lumière merveilleuse, dans un paysage saisi par le givre, aux couleurs tantôt dorées, tantôt roses d'un soleil rasant.

Et la partie scientifique?

Un très beau programme, dont nous retiendrons tout particulièrement la dermatologie arctique... Présentation de notre spécialité au Groenland. L'eczéma atopique y fleurit, favorisé par la xérose cutanée, à côté de toute la pathologie du froid. Doit-on s'en protéger? Crème ou onguent? Rien du tout, d'après les études de l'armée finlandaise. Les crèmes en s'évaporant - accentuent l'effet du froid, les onguents donnent une fausse impression de bien-être et les gelures s'installent à l'insu de leur victime... Et ne vous réchauffez pas n'importe comment... Au Groenland, les maladies vénériennes sont 10 fois plus fréquentes que dans les pays scandinaves...

\section{Et l'EADV?}

Une révolution aussi... De nouveaux statuts, enfin démocratiques. L'EADV qui refusait la Suisse en 1989 (souvenezvous de mes coups de gueule de l'époque...) déplace son siège de Luxembourg à Lugano! Il fallait venir au nord du cercle polaire pour voir nos relations enfin dégelées!

Une raison de plus pour ceux qui n'en seraient pas encore membres de rejoindre l'EADV!

\section{A.-A. Ramelet, Lausanne}

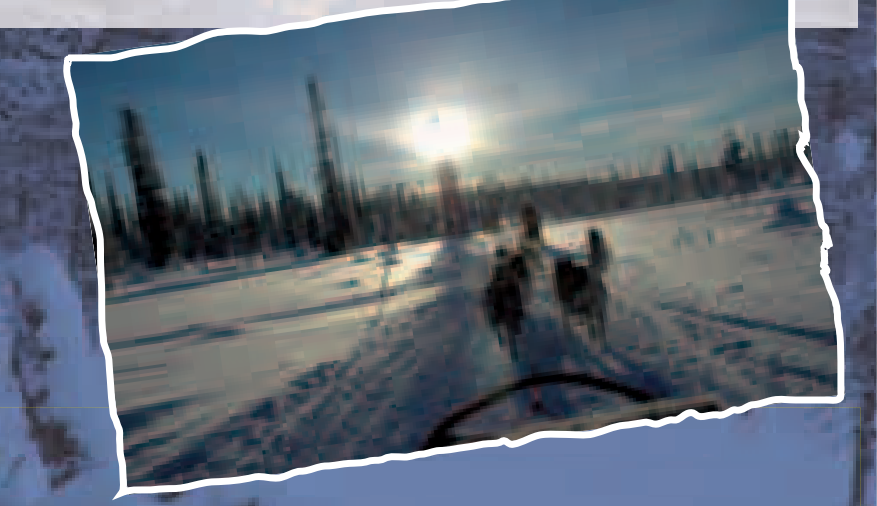

\section{Effectuer le traitement topique de Caryolysine ${ }^{\circledR}$ à domicile en toute sécurité}

\section{Marche à suivre détaillée pour le patient et/ou la personne appliquant le traitement à domicile}

\author{
Rédigé par: $\quad$ M. Ackermann, L. Bigler, L. Blal, \\ S. Devos, D. Conan, L. French, \\ L.Z. Kaestli, N. Vogt \\ Avec la collaboration de C. Sierro et \\ S. Radji de SOS Pharmaciens \\ Relu par: $\quad$ C. Beguin, N. Bessire, K. Bullani, \\ P. Bonnabry, P. Brennenstuhl, \\ P. Chopard, J. Desmeules, C. Prins
}

Approuvé par: Prof. J.-H. Saurat

\section{Les 5 points à retenir:}

1) Pour effectuer le traitement à domicile, il faut avoir reçu un enseignement par une infirmière spécialisée en dermatologie.

2) La Caryolysine ${ }^{\circledR}$ est un produit qui nécessite certaines protections particulières.

3) Les femmes enceintes et les enfants ne doivent pas entrer en contact avec la Caryolysine.

4) La Caryolysine s'utilise uniquement diluée.

5) Ne jetez pas la Caryolysine dans le lavabo, mais appliquez la procédure d'élimination des déchets des cytotoxiques

Des applications topiques de Caryolysine ont été prescrites par votre dermatologue.

Ce document contient toutes les informations dont vous avez besoin pour réaliser ces applications à votre domicile en toute sécurité.

\section{Personnes habilitées à appliquer le traitement}

- Les premières applications sont effectuées par une infirmière. Le traitement peut être ensuite poursuivi à domicile par le patient, une personne de son entourage ou une infirmière à domicile. Il est très important que la personne qui applique le traitement à domicile ait été formée par une infirmière spécialisée en dermatologie.

- Une femme enceinte et/ou un enfant ne doivent ni être présents dans la pièce lors de l'application, ni appliquer la solution, ni effectuer le nettoyage après l'application. 


\section{Nouvelles des cliniques \\ Neues aus den Kliniken}

\section{Précautions à respecter}

Le produit est irritant. Pour une utilisation sûre, certaines précautions sont nécessaires:

- Manipuler la Caryolysine qu'une fois la tenue de protection revêtue et le sol protégé

- Eviter l'inhalation du produit

- A utiliser uniquement dilué

- Appliquer le traitement sur une peau bien sèche; attendre $1 \mathrm{~h}$ après un bain ou une douche

- Ne pas se laver durant les $3 \mathrm{~h}$ suivant l'application de la Caryolysine

- Se doucher le soir avant de se coucher

- Laver le linge en contact avec la peau séparément de celui des autres membres de la famille

\section{Conditions pour effectuer ce traitement à domicile}

- Posséder une pièce sans tapis et qui puisse être aérée si possible

- Le traitement ne se fait pas dans la cuisine

Une ordonnance vous sera délivrée par votre médecin afin d'avoir à votre disposition le matériel nécessaire à la préparation du produit, ainsi que le matériel de protection.

Face à d'éventuelles difficultés rencontrées, il vous est toujours possible de nous contacter; nous répondrons à toutes vos interrogations.

Nos coordonnées:

- Service ambulatoire (8-18 h)

- Service hospitalier (24 h/24 h)

Tél. 0223729452 Tél. 0223723311

En cas d'arrêt de traitement, rendre toutes les boîtes entamées ou entières à votre dermatologue ou à votre pharmacie

\section{Informations sur le produit}

\section{Médicament:}

Caryolysine existe sous forme d'ampoules à $10 \mathrm{mg}$

DCI (Dénomination Commune Internationale):

chlorméthine

\section{Catégorie thérapeutique:}

Cytostatique/alkylant

Quelles sont les maladies qui peuvent être traitées avec ce médicament?

- Traitement du lymphome cutané épidermotrope (mycosis fongoïde)

- Traitement local du psoriasis de l'adulte

\section{Que faire en cas d'oubli?}

Ne pas rattraper une application oubliée. Pour avoir une meilleure réponse, il est important de respecter la fréquence prescrite par le dermatologue.
Quels sont les effets secondaires possibles?

- Environ 10\% des patients peuvent développer une sensibilisation après 21-30 jours: ceci se manifeste par des démangeaisons, irritation, brûlure ou un eczéma de contact sur les surfaces où la solution a été appliquée. Ces manifestations doivent faire arrêter l'application du produit et devront être signalées immédiatement à votre dermatologue (ou à un médecin de garde en précisant que vous prenez de la Caryolysine en application topique ainsi que les autres médicaments).

- Une irritation locale est fréquente, s'atténuant lors de l'espacement des applications. Dans ce cas, le traitement peut se poursuivre.

\section{Mise en garde}

- Il est recommandé de ne pas prendre d'autres médicaments, même en automédication sans en informer préalablement votre médecin ou votre pharmacien.

- Ne pas prendre de bain avec du bicarbonate de sodium, car il inactive le traitement.

- La Caryolysine augmente la sensibilité au soleil. Utilisez une crème solaire de protection 15 ou plus.

\section{Grossesse et allaitement}

Toutes les femmes en âge de procréer doivent utiliser des méthodes contraceptives afin d'éviter une grossesse pendant la durée du traitement. En cas de grossesse, veuillez arrêter le traitement de Caryolysine et en informer votre dermatologue.

Comment conserver ce médicament?

- Tenir ce médicament, la solution préparée et les déchets hors de portée des enfants.

- Les ampoules sont à conserver à température ambiante. Ne pas les utiliser au-delà de la date d'expiration.

- Les ampoules entamées ainsi que la solution préparée sont instables. Elles ne doivent pas être conservées.

Faut-il observer des précautions particulières?

- En cas de contact accidentel avec la peau saine, les muqueuses ou les yeux:

- Rincez la partie concernée (peau ou œil) à l'eau froide du robinet pendant au moins 5 min.

- En cas de projection dans les yeux, consultez ensuite un ophtalmologue.

- En cas de déversement accidentel sur une surface:

$\rightarrow$ Appliquez ces précautions en cas d'éclaboussement ou casse de l'ampoule

- Mettez le masque, les lunettes de protection, la surblouse, les gants en vinyle et par-dessus la paire de gants de chimioprotection.

- Epongez avec des chiffons absorbants.

- Jetez les chiffons dans le seau à déchets cytotoxiques.

- Lavez et rincez avec beaucoup d'eau.

- Mettez le matériel utilisé dans le sac en plastique et jetez-le dans le seau. 


\section{Informations sur le mode d'application et les précautions à prendre}

Les premières applications de Caryolysine sont faites sous la supervision d'une infirmière spécialisée qui donnera un enseignement sur le mode d'application à suivre (pour vous ou la personne de votre entourage qui effectuera ce soin).

$\rightarrow$ Il est important de respecter les recommandations de sécurité qui suivent.

\section{Déroulement du soin}

\section{Préparatifs avant l'application}

- Aérez la pièce: $\rightarrow$ Si possible pendant le traitement

$\rightarrow$ Systématiquement après le traitement

- Posez un champ de protection sur le sol à l'endroit où vous allez vous tenir pour faire le traitement. Veillez à avoir de la place dégagée et libre autour de vous: évitez de faire le traitement juste à côté de votre lit ou d'un fauteuil; en cas de traitement à proximité d'un meuble non lavable, recouvrez-le avec un champ de protection.

- Choisissez une surface rigide, libre de tout objet et facile à nettoyer et posez-la sur la protection du sol et protégez-la avec un autre champ de protection.

- Posez un seau pour récupérer les déchets cytotoxiques sur ce champ de protection.

- En cas de traitement des plantes des pieds, préparez un siège sur la protection du sol et couvrez-le aussi d'une protection.

- Posez sur la surface choisie:

1 flacon d'eau distillée $100 \mathrm{ml}$

1 pipette en plastique

1 ampoule de Caryolysine

1 tube crème Elocom ${ }^{\circledR}$

1 paire de gants de chimio-

protection

1 paire de gants vinyle

Des compresses

\section{1 masque}

1 paire de lunettes de protection

1 surblouse

1 récipient vide

Des champs de protection

Sac en plastique

Seau

\section{Mesures de protection personnelles}

- Déshabillez-vous et placez-vous sur la protection posée au sol.

- La personne qui est traitée et la personne qui applique le traitement mettent un masque, les lunettes de protection, les gants en vinyle. La personne qui applique le traitement met une paire de gants de chimioprotection par-dessus les gants en vinyle.

- Lorsque le traitement est appliqué par une tierce personne, cette dernière met une surblouse de protection.

Modalité d'application du traitement

a) Dilution de la Caryolysine

- Versez $50 \mathrm{ml}$ d'eau du flacon dans le récipient vide ou dans le lavabo.

$\rightarrow$ Il reste $50 \mathrm{ml}$ d'eau dans le flacon.

- Cassez l'ampoule de Caryolysine $10 \mathrm{mg}(2 \mathrm{ml})$ en l'entourant d'une compresse.

- Prélevez le contenu de l'ampoule avec la pipette.

- Injectez-le lentement dans le flacon d'eau distillée.

$\rightarrow$ Bouchonnez le flacon et mélangez bien.
- Jetez la pipette, l'ampoule vide et la gaze dans le sac en plastique.

b) Application de la Caryolysine

- Prenez une compresse et imbibez-la de la solution diluée en appliquant la compresse contre le goulot de la bouteille.

- Passez la compresse mouillée, 1 fois, sur les zones à traiter, sans frotter, en commençant par le haut du corps. Remouillez régulièrement la compresse pendant l'application.

$\rightarrow$ NE PAS traiter: le visage, les parties génitales et les plis

$\rightarrow$ A traiter dans tous les cas: toute la surface de la peau, y compris les fesses même s'il n’y a pas de lésions (sauf avis médical contraire)

$\rightarrow$ A traiter sur ordre médical: les plantes des pieds et les mains. Si vous devez traiter les plantes des pieds, asseyez-vous sur le siège protégé pour l'application et laissez sécher les pieds sans les poser à plat sur le sol.

- Après l'application, restez debout et attendez que votre peau soit sèche (environ $10 \mathrm{~min}$ ).

- Bouchonnez le flacon qui contient la solution de Caryolysine diluée.

- Mettez le flacon, les compresses mouillées et les gants bleus de chimioprotection dans le sac en plastique.

- Gardez encore les gants en vinyle.

- Appliquez l'Elocom sur les surfaces traitées. Elle améliore la tolérance et diminue les effets secondaires du traitement.

- Attendez 5 min.

- Jetez la protection protégeant la surface de travail et celle du sol dans la poubelle ménagère.

- Enlevez et jetez les gants en vinyle dans le sac en plastique.

- Rhabillez-vous.

- Aérez la pièce.

- Lavez-vous les mains au savon.

- Le soir avant le coucher ou au plus tôt $3 \mathrm{~h}$ après le traitement, prenez une douche et savonnez-vous sur tout le corps.

- L'utilisation de lotion hydratante pendant la journée peut être utile en cas de peau sèche.

\section{Gestion des déchets}

Ne jetez pas le produit pur ou dilué dans le lavabo. Mettez dans le sac en plastique tous les déchets contaminés, qui sont:

- Le flacon d'eau dans lequel la Caryolysine a été mise

- La pipette

- Les compresses

- L'ampoule de Caryolysine

- Toutes les paires de gants

- Les champs de protection

- Le masque et les lunettes

- La surblouse

$\rightarrow$ Ce sac est à mettre dans le seau cytotoxique. Lorsque ce dernier est plein fermez le couvercle hermétiquement et ramenez-le lors de la prochaine consultation chez votre dermatologue. 